Related content

\title{
Spontaneous electron emission from a cold surface
}
Interplay of Tomonaga-Luttinger liquids and superconductive phase in carbon nanotubes
J. Haruyama, J. González, E. Perfetto et a.
- Slip avalanches in a fiber bundle model Z. Halász and F. Kun
- Universality class of fiber bundles with strong heterogeneities R. C. Hidalgo, K. Kovács, I. Pagonabarraga et al.

View the article online for updates and enhancements.

Recent citations

- The IceCube Neutrino Observatory:
$\frac{\text { instrumentation and online systems }}{\text { M.G. Aartsen et al }}$
- Desian and construction of the
$\frac{\text { MicroBooNE detector }}{\text { R. Acciarri et al }}$
- $\frac{\text { IceCube sensitivity for low-energy }}{\text { neutrinos from nearby supernovae }}$
R. Abbasi et al




\title{
Spontaneous electron emission from a cold surface
}

\author{
H. O. MEYER ${ }^{(a)}$ \\ Physics Department, Indiana University - Bloomington, IN 47405, USA
}

received 1 February 2010; accepted in final form 17 February 2010

published online 26 February 2010

PACS $85.60 . \mathrm{Ha}$ - Photomultipliers phototubes and photocathodes

PACS 79.75.+g - Exoelectron emission

\begin{abstract}
At cryogenic temperature, the dark rate in a photomultiplier is caused by single electrons, emitted spontaneously from the cathode surface. This "cryogenic" dark rate increases with decreasing temperature down to at least $4 \mathrm{~K}$. The average event rate is proportional to the area of the emitting surface and insensitive to the electric field at that surface. The electrons are emitted in bursts. The bursts are distributed randomly in time, but the events within a burst are highly correlated. The burst durations are distributed according to a power law. As the temperature decreases, the rate of bursts, as well as the number of events per burst, increase. The observed time distributions are indicative of a trap mechanism. So far, there is no physics explanation of the observed phenomenon.
\end{abstract}

Copyright (C) EPLA, 2010

Introduction. - At room temperature, the pulse rate observed in a photomultiplier in the absence of light (dark rate) is mostly due to thermionic emission of electrons. When the temperature is lowered, this dark rate decreases according to Richardson's law. However, below about $220 \mathrm{~K}$ the dark rate levels off, and with further cooling rises monotonically, at least down to a temperature of $4 \mathrm{~K}$.

This phenomenon, which we call "cryogenic" electron emission, has been known to exist for some time $[1,2]$. The present paper contains a complete experimental characterization of the dark rate at low temperature. The effect is caused by the emission of single electrons and can thus be observed in photomultipliers, which provide the necessary large-gain current amplification. Cryogenic emission has some remarkable features, and is interesting in its own right; it should not be dismissed as an exotic feature of a specialized device.

Average emission rate. - Our measurements of the average dark rate as a function of temperature are shown in fig. 1. The data are obtained by placing the photomultiplier inside an evacuated container, which is then submerged in liquid nitrogen or helium. Cooling is by radiation, initially by about $30 \mathrm{~K} / \mathrm{h}$. Equilibrium at $80 \mathrm{~K}$ is established after about a day. The temperature is measured by a silicon diode sensor, coupled to the glass surface at the cathode. Pulses at the anode are discriminated at about one-third of the charge that corresponds to a single electron. Practically no events are observed

\footnotetext{
(a) E-mail: meyer1@indiana.edu
}

when the threshold is set above the single-electron peak, or when the cathode potential is set to that of the first dynode. This demonstrates that cryogenic dark events are caused by single electrons, emitted from the photocathode. Our data extend down to $4 \mathrm{~K}$, and have been obtained with two different Hamamatsu R7725, 5-cm-diameter photomultipliers. Data sets acquired during cool-down, or during warm-up of the apparatus agree with each other, demonstrating that cryogenic emission does not depend on the temperature evolution prior to the measurement. Also shown in fig. 1 are the sparse data from previous measurements [3,4]. All data in fig. 1 have been obtained with photomultipliers with bi-alkali cathodes (K-Cs-Sb) on a thin platinum backing. This backing is needed below $150 \mathrm{~K}$ to counteract the loss of conductivity of the cathode material.

The cathode areas of the various multipliers represented in fig. 1 range over one and a half orders of magnitude, yet, the dark rates per cathode area lie near a common locus that can be expressed by $r_{c}=G \cdot A \cdot \exp \left(-T / T_{c}\right)$ (solid line), where $A$ is the cathode area, $T_{c}=100 \mathrm{~K}$, and $G=5 \mathrm{~cm}^{-2} \mathrm{~s}^{-1}$ is the emission rate per cathode area at $0 \mathrm{~K}$. Thus, the cryogenic emission rate scales with the area of the emitting surface.

When the operating voltage is varied, the cryogenic dark rate changes little, if at all (open symbols in fig. 2), demonstrating that cryogenic emission is not sensitive to the electric field at the cathode surface. This is different from thermionic emission, which increases by an order of magnitude as the voltage is raised by about 25\% (solid symbols in fig. 2). 


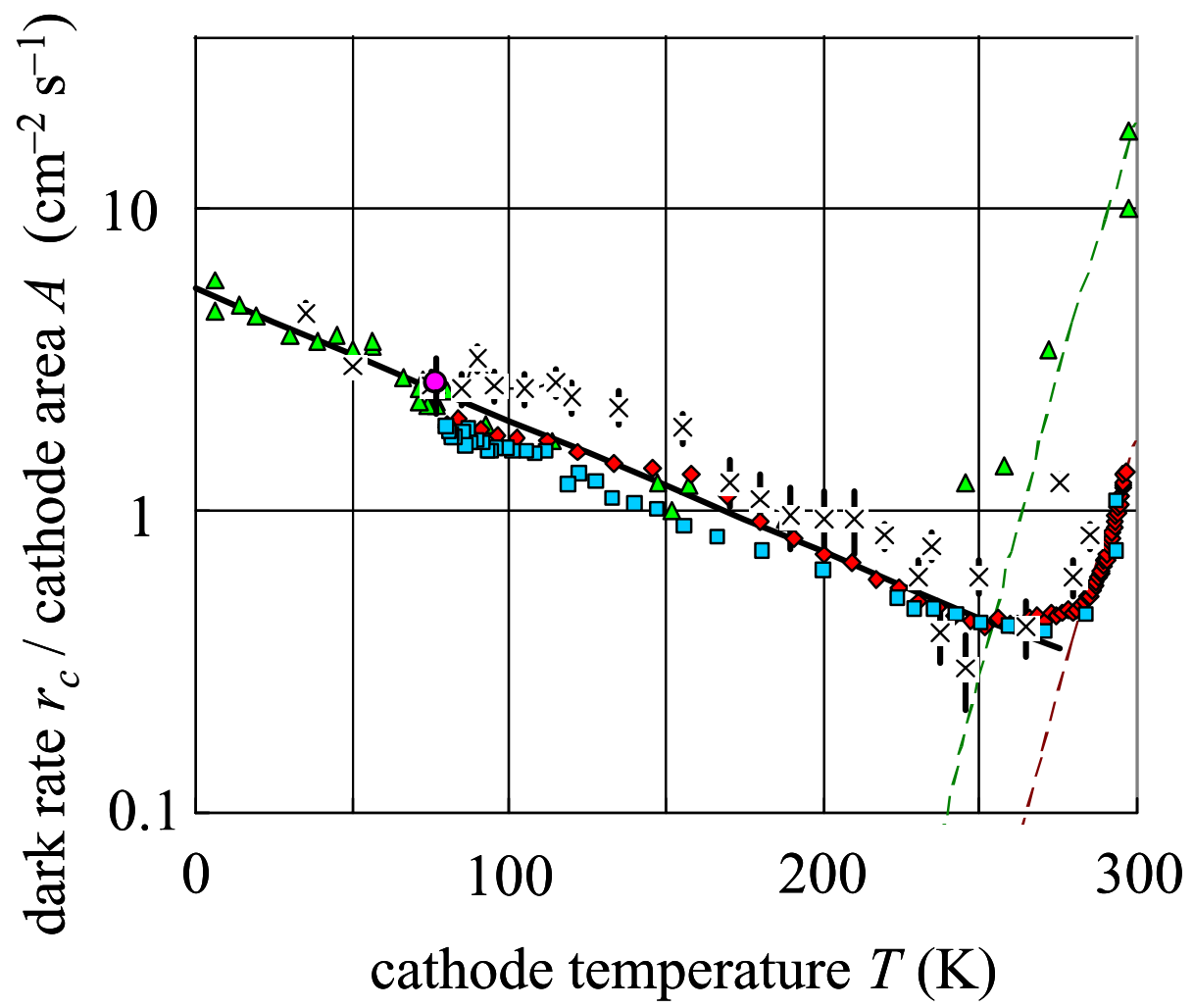

Fig. 1: Dark rate per cathode area observed with two Hamamatsu R7725 tubes (cathode area $A=17 \mathrm{~cm}^{2}$ ). Tube 1 was cooled to $4 \mathrm{~K}$ (triangles), and tube 2 was cooled to $81 \mathrm{~K}$ (squares) and then warmed up again (diamonds). The dashed lines indicate the temperature dependence expected for thermionic emission (Richardson's law) for these two tubes. Also shown are measurements with a Hamamatsu R5912 (crosses, $A=335 \mathrm{~cm}^{2}$, ref. [3]), and with an ETL 9357FLA (circle, $A=430 \mathrm{~cm}^{2}$, ref. [4]). The solid line marks the exponential function mentioned in the text.

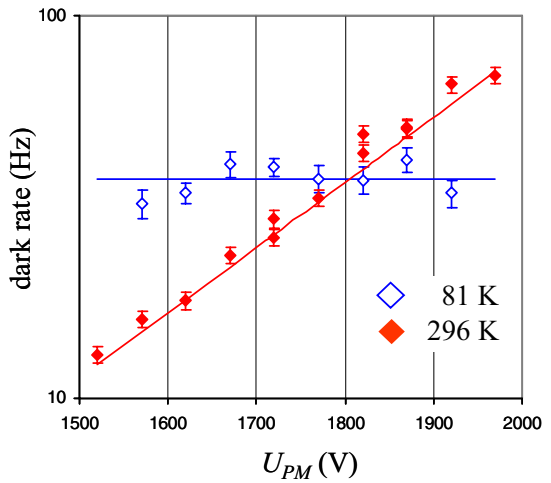

Fig. 2: (Colour on-line) Average dark rate as a function of the photomultiplier voltage, observed with a Hamamatsu R7725 tube. At $81 \mathrm{~K}$ or $296 \mathrm{~K}$, cryogenic or thermionic emission dominates, respectively.

It is known that the thermionic emission rate in different photomultipliers may vary a lot, even when they are of the same model (e.g., triangles and diamonds at $300 \mathrm{~K}$ in fig. 1). On the other hand, cryogenic emission (per area) seems to be insensitive to parameters that one expects to vary with different manufacturers and operating conditions (such as radioactive contaminants, magnetic field, mechanical stresses, vibration, etc.). It is true that all photomultipliers represented in fig. 1 have bi-alkali (K-Cs-Sb) cathodes on a thin platinum backing, but cryogenic emission (that is, its onset at $T>150 \mathrm{~K}$ ) has also been observed with tri-alkali (Sb-Na-K-Cs) cathodes, and without a metal backing $[1,2]$.

Time correlations. - Cryogenic dark events do not occur randomly. In the following, we investigate how these events are distributed in time. To this effect, we have measured the time of occurrence of each event in a given sample. This is achieved by timing the spacing $\Delta t_{n}(n=1, \ldots, N)$ between subsequent pulses to within $1 \mu \mathrm{s}$, using a gated counter and a $1-\mathrm{MHz}$ clock. The sample evaluated in the following has been acquired at a temperature of $T=81 \mathrm{~K}$, during a time $t_{N}=857 \mathrm{~s}$, and contains $N=30391$ intervals.

The distribution of the intervals $\Delta t_{n}$ is shown in fig. 3 . It consists of an exponential part (marked by a solid line), and a spike at small $\Delta t$. For uncorrelated pulses there would be no spike. We define as "short intervals" those within the spike, using the arbitrary cutoff $\Delta t<7 \mathrm{~ms}$, shown as a dashed line in the insert of fig. 3. Most of the intervals in our sample are in fact "short" (71\%), and 


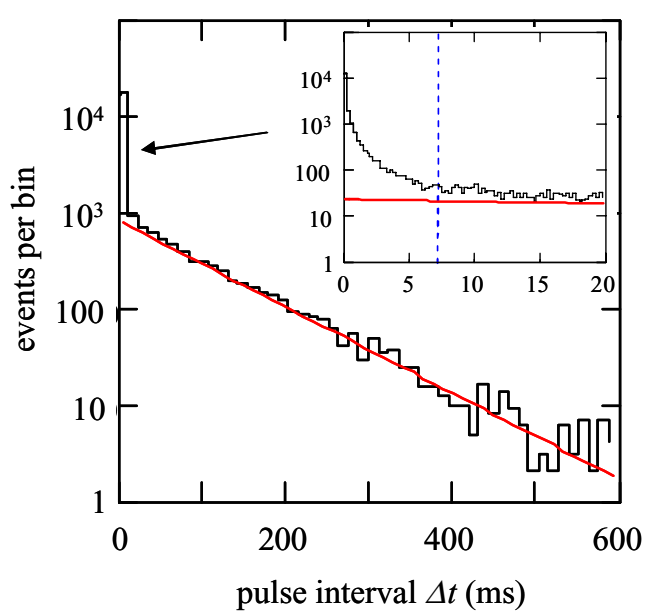

Fig. 3: (Colour on-line) Distribution of intervals between dark events observed at $T=81 \mathrm{~K}$. The insert shows the range of small $\Delta t$ with finer binning. The solid line is a fit to the exponential part of the distribution (same in main graph and insert). The dashed line divides the data into "short" and "long" intervals.

of those most are much shorter than $7 \mathrm{~ms}$, which makes the definition of "short" insensitive to the cutoff value.

Bursts. - Let us define a "burst" as an uninterrupted sequence of "short" intervals. Then, our sample contains $N_{s}=21411$ short intervals and $M=3711$ bursts. The number of intervals in a given burst we call "size". The average burst size is then $\langle L\rangle=N_{s} / M$. The number $F_{L}$ of bursts of a given size $L$ is shown in fig. 4 . This distribution is well described by the solid line in fig. 4, which is calculated from

$$
F_{L}=M \cdot\langle L\rangle \frac{1-q}{q} \cdot \frac{q^{L}}{L} .
$$

The constant $q$ is actually a function of $\langle L\rangle$, which can be found by setting the sum of $F_{L}$ over all $L$ equal to the number $M$ of bursts. Thus, the shape of the burst size distribution is determined by the average burst size $\langle L\rangle$. That the short intervals are, in fact, correlated is illustrated by the dashed line, which would result if the same fraction $N_{s} / N=0.71$ of short intervals were distributed randomly throughout the sample.

The smallest burst $(L=1)$ is a pair of pulses separated by a short interval. "Single events" with $L=0$ then are pulses that are preceded and followed by a long interval $\left(F_{0}\right.$ is shown as an open circle in fig. 4$)$. These events make up the exponential tail in fig. 3 .

Let $\Delta \tau_{m}$ be the time between consecutive bursts, marked by the first pulse in the burst. One finds that the distribution of intervals $\Delta \tau_{m}$ is in good agreement with a simple exponential function, $\exp \left(-\Delta \tau \cdot M / t_{N}\right)$, where $M / t_{N}=4.2 \mathrm{~Hz}$ is the average burst rate. This indicates that bursts occur independently of each other.

We define the duration $D_{L}$ of a burst of size $L$ as the time between the first and the last pulse in a burst, averaged over all bursts of that size. The distribution of

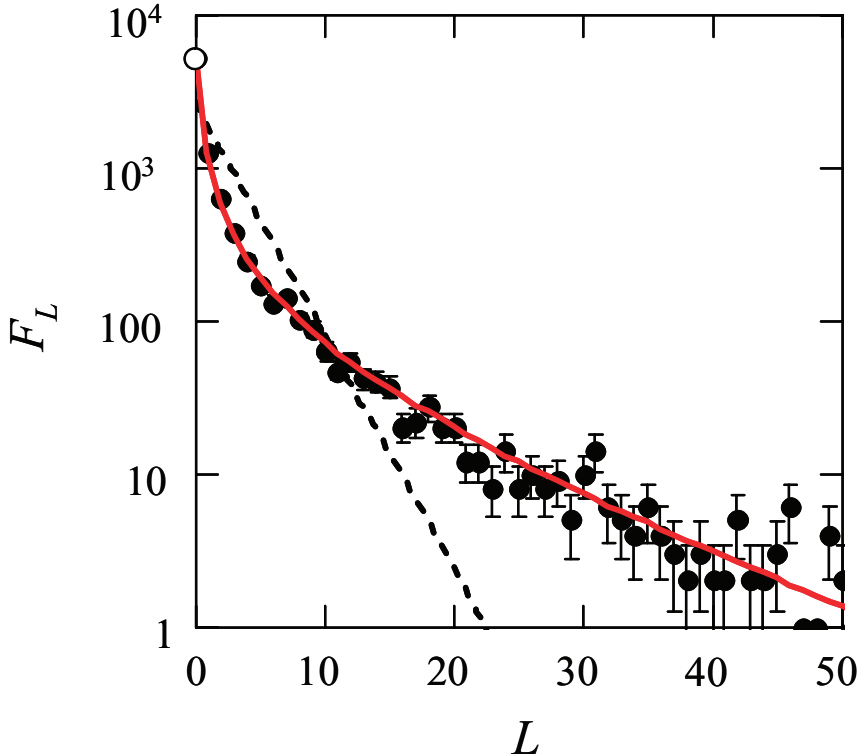

Fig. 4: (Colour on-line) Burst size distribution $F_{L}$ at $T=81 \mathrm{~K}$. The number of single pulses is shown as open circle. The average burst size is $\langle L\rangle=5.77$. The solid line is calculated from eq. (1) using $q=0.943$. The dashed line shows the distribution that would result if the same number of short and long intervals were arranged randomly.
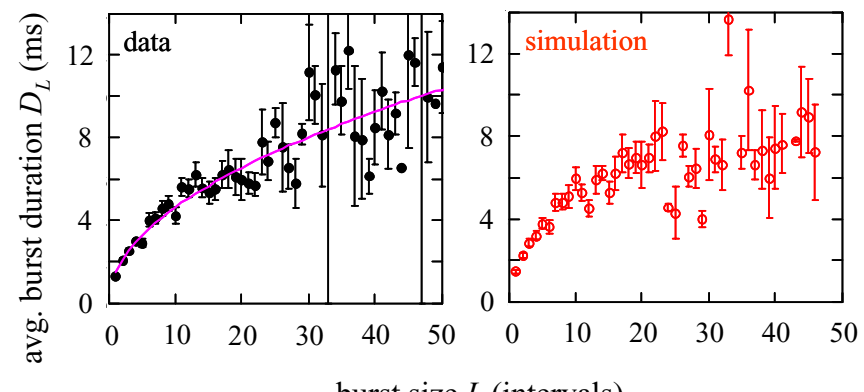

burst size $L$ (intervals)

Fig. 5: (Colour on-line) Average bursts duration $D_{L}$ vs. burst size $L$. The left panel shows the data and the right panel is generated by the model described in the text. The solid line is proportional to $L^{\frac{1}{2}}$.

$D_{L}$ is shown in fig. 5. The solid line is proportional to $L^{\frac{1}{2}}$, indicating that the burst duration distribution obeys a power law.

We now turn to a study of the remarkable pattern of event spacings within a given burst. Let us define $\delta \tau_{m, \ell}$ as the $\ell$-th interval of the $m$-th burst. Picking a certain burst size arbitrarily (here, $L=15$ and 25 ), we plot in fig. 6 the intervals $\delta \tau_{m, \ell}$ vs. their placement $\ell$ in the burst. For instance, the symbols in the first column in each panel mark the time between the first and the second pulse for all recorded bursts of size $L$. The duration of the last interval appears in the $L$-th column. From the figures, it is obvious that the intervals between pulses in a burst are growing as the burst progresses, from about $3 \mu \mathrm{s}$ in the beginning 


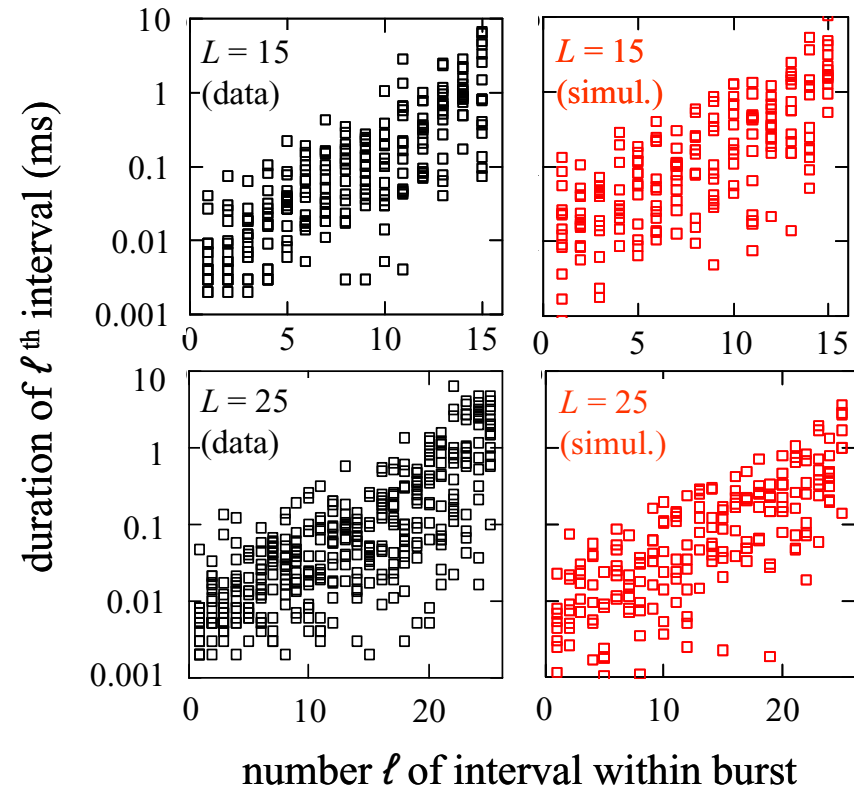

Fig. 6: (Colour on-line) Time intervals $\delta \tau_{m, \ell}$ for all bursts of a given size $L$ vs. the place $\ell$ within the burst. Measurements for burst size $L=15$ and 20 are shown on the left, the corresponding simulations (see text) on the right.

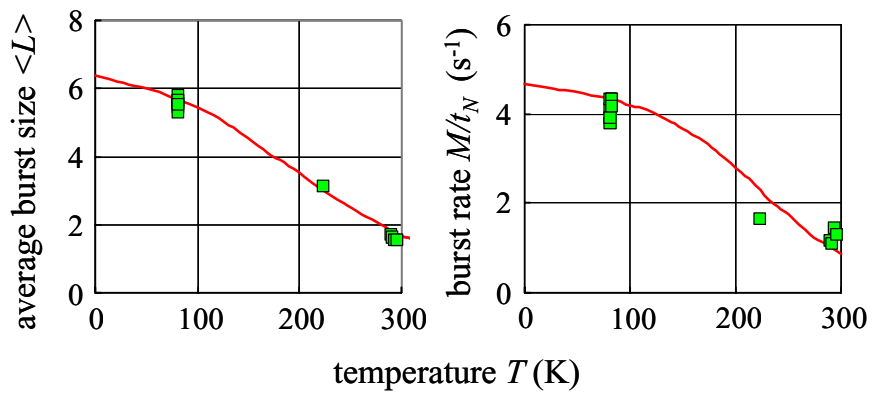

Fig. 7: (Colour on-line) Average burst size $\langle L\rangle$ and burst rate as a function of temperature. The lines are generated by the model described in the text.

to about $3 \mathrm{~ms}$ at the end of the burst. Apparently, this behavior is very similar for bursts of different size.

Temperature dependence. - Up to this point, all data shown have been collected at $81 \mathrm{~K}$. We now turn to the temperature dependence. In fig. 7 the average burst size $\langle L\rangle$ and the burst rate $M / t_{N}$ are shown for three temperatures, including room temperature. We have seen earlier that these two parameters are sufficient to describe the burst size distribution. It is important to note that an increase in the cryogenic dark rate is due to both, an increase in the burst rate, as well as the burst size. The solid line in fig. 7 is deduced from a model, which will be discussed in the following.

Empirical trap model. - The peculiar, nonstochastic time distribution observed for cryogenic dark events is indicative of a trapping mechanism. To demonstrate this, we postulate a heuristic trap, which is initially filled with $k_{0}$ electrons. An electron either exits the trap (to be observed as a dark event), or it is recombined. The rate $R$ at which the trap is emptying is proportional to the present content $k$, and assumed to be

$$
R=k \cdot\left[\varepsilon \cdot\left(1+\gamma\left(k / k_{0}\right)^{4}\right)+\alpha \cdot \exp \left(T / T_{0}\right)\right] .
$$

The emission rate (the first term) is described by a constant $\varepsilon$, modified by a factor that takes into account that emission may be enhanced for a full trap because of electrostatic repulsion. The recombination rate is described by a constant $\alpha$, modified by an exponential function of the temperature. Recombination competes with emission. The fact that the observed emission rate grows with decreasing temperature is now a consequence of recombination becoming less important. The model has four adjustable parameters, $\varepsilon, \gamma, \alpha$, and $T_{0}$.

The calculation starts with a number of traps, filled in such a way that the distribution of eq. (1) is reproduced. For each trap, the time of the next electron event is determined randomly (from the present rate $R$ ), and it is decided randomly whether the electron is absorbed or emitted (from the present ratio of the two terms in eq. (2)), then the trap count $k$ is lowered by one. This is repeated until the trap is empty. Keeping track of the times between emitted electrons, the burst duration $\left(D_{L}\right)$ distribution, and a list of simulated $\Delta t$ are accumulated. The parameters have been fixed to $\varepsilon=300 \mathrm{~s}^{-1}, \gamma=9$, $\alpha=20 \mathrm{~s}^{-1}$, and $T_{0}=50 \mathrm{~K}$. In the simulated results shown in fig. 6 , the emission rate $\varepsilon$ sets the overall timescale, while $\gamma$ can be used to fine-tune the slope of the bands. The model with its fixed parameters is able to generate the simulations in fig. 6 for both burst sizes, as well as the $D_{L}$ distribution in fig. 5 .

The dependence on temperature is caused by the absorption term (second term in eq. (2)) with two parameters and a functional dependence on $T$, which has been chosen to reproduce the data in fig. 7 . However, it is still remarkable that the model simultaneously explains both, burst rate and burst size (solid lines). In addition, one finds that burst size distributions $F_{L}$, observed at different temperature, are also reproduced by the model.

Summary and conclusions. - In this paper, we have documented the properties of spontaneous emission of electrons from the cathode of a photomultiplier operated at low temperature, with no light. In this process, which we call "cryogenic" emission, single electrons are ejected. Let us summarize our findings.

Cryogenic emission dominates below $200 \mathrm{~K}$. The time-averaged rate depends exponentially on (minus) the temperature, and is highest at the lowest temperature measured $(4 \mathrm{~K})$. The rate does not depend on the evolution of the temperature prior to the measurement, and is insensitive to the electric field at the emitting surface. The observed average event rate is proportional to the area of the emitting surface. Apart from that, it is insensitive to variations such as one might 
expect in photomultipliers from different manufacturers, studied by different scientists under different operating conditions.

The electrons are emitted in bursts. The bursts themselves occur randomly, but within a burst the events are highly correlated in that their mean separation steadily increases from about $3 \mu \mathrm{s}$ to about $3 \mathrm{~ms}$ as the burst evolves. This behavior is the same for bursts of any size. The burst duration distribution follows a power law. As the temperature decreases, both the rate of bursts and their size increase.

Our heuristic model suggests that the observed time distribution of dark events indicates a trap mechanism, but leaves unexplained what physical processes are responsible for the creation, filling and emptying of the traps, what causes ionization and recombination, and what explains the burst size distribution. Perhaps, there is a connection to traps in semiconductors for which there is an extended literature (for example, see $[5,6]$ ).

A process that becomes more probable with decreasing temperature is very unusual. Such abnormal behavior has been ascribed to a "decrease of the lattice energy upon cooling of the emitting material" [4]. In another instance of abnormal temperature dependence (in this case the $1 / f$ noise in a bismuth sample) the suggested explanation is in the "defect-mediated quantum interference of conduction electrons" [7]. In our empirical model, discussed earlier, the abnormal behavior is due to competition with a process with a normal energy dependence. At this stage, these are just hints that may or may not lead to an understanding of cryogenic emission.

The properties of cryogenic emission are inconsistent with any of the spontaneous emission processes known, such as thermal emission, field emission, radioactivity, or penetrating radiation, including cosmic rays. At this time, regrettably, a quantitative explanation of the observed characteristics of cryogenic emission in terms of known physics is still eluding us.

We thank Prof. I. Sick, University of Basel, for his insightful advice, and D. BAXTER, T. RINCKEL, T. Sulanke, D. Sprinkle, and the machine shop crew at the Indiana University Physics Department for their support of this work.

\section{REFERENCES}

[1] Rodman J. P. and Smith H. J., Appl. Opt., 2 (1963) 181.

[2] Gadsden M., Appl. Opt., 4 (1965) 1446.

[3] Nikkel J. A., Lippincott W. H. and McKinsey D. N., J. Instrum., 2 (2007) 11004.

[4] Ankowski A. et al., Nucl. Instrum. Methods. A, 556 (2006) 146.

[5] Aurel F. D. et al., Appl. Phys. Lett., 74 (1999) 407.

[6] Chen I. C., Holland S. and Hu C., J. Appl. Phys., 61 (1987) 4544.

[7] Birge N. O., Golding B. and Haemmerle W. H., Phys. Rev. Lett., 62 (1989) 195. 